\title{
ORNL/CP-94692
}

$$
\text { CONF- } 970622--
$$

\section{FRONTIERS OF NUCLEAR STRUCTURE}

\section{Witold Nazarewicz}

Department of Physics, University of Tennessee, Knoxville, TN 37996, U.S.A. Physics Division, Oak Ridge National Laboratory*, Oak Ridge, TN 37831, U.S.A. Institute of Theoretical Physics, Warsaw University, PL-00681, Warsaw, Poland

\section{RECEIVED \\ OCT 271997 \\ OST I}

\section{Proceedings of}

6th International Conference on Nucleus-Nucleus Collisions

Gatlinburg, TN

June 2-6, 1997

(to be published in Nuclear Physics A)

\section{6}

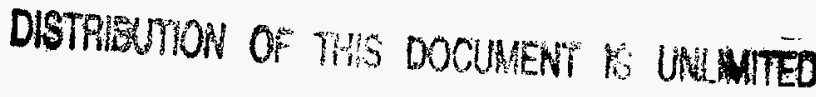
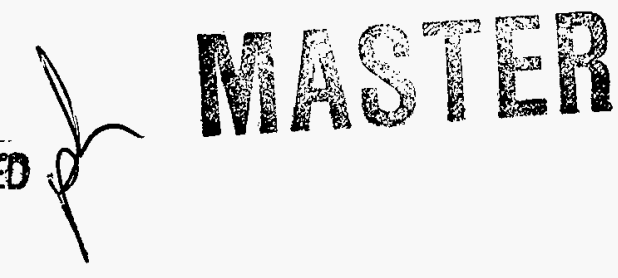

*Managed by Lockheed Martin Energy Research Corp. under contract DE-AC0596OR22464 with the U.S.D.O.E.

"The submitted manuscript has been authored by a contractor of the U.S. Government under contract No. DE-AC05-960R22464. Accordingly, the U.S. Government retains a nonexclusive, royalty-free license to publish or reproduce the published form of this contribution, or allow others to do so, for U.S. Government purposes." 


\section{DISCLAIMER}

This report was prepared as an account of work sponsored by an agency of the United States Government. Neither the United States Government nor any agency thereof, nor any of their employees, make any warranty, express or implied, or assumes any legal liability or responsibility for the accuracy, completeness, or usefulness of any information, apparatus, product, or process disclosed, or represents that its use would not infringe privately owned rights. Reference herein to any specific commercial product, process, or service by trade name, trademark, manufacturer, or otherwise does not necessarily constitute or imply its endorsement, recommendation, or favoring by the United States Government or any agency thereof. The views and opinions of authors expressed herein do not necessarily state or reflect those of the United States Government or any agency thereof. 


\section{Frontiers of Nuclear Structure}

Witold Nazarewicz ${ }^{\text {a* }}$

a Department of Physics, University of Tennessee, Knoxville, TN 37996, U.S.A.

Physics Division, Oak Ridge National Laboratory, Oak Ridge, TN 37831, U.S.A.

Institute of Theoretical Physics, Warsaw University, PL-00681, Warsaw, Poland

Current developments in nuclear structure at the "limits" are discussed. The studies of nuclear behavior at extreme conditions provide us with invaluable information about the nature of the nuclear interaction and nucleonic correlations at various energy-distance scales. In this talk, frontiers of nuclear structure are briefly reviewed from a theoretical perspective, mainly concentrating on medium-mass and heavy nuclei.

\section{INTRODUCTION}

The atomic nucleus is a fascinating many-body system bound by strong interaction. The building blocks of a nucleus, protons and neutrons, are themselves composite aggregates of quarks and gluons governed by quantum chromodynamics (QCD) - the funclamental theory of strong interaction. But even if one simplifies the problem by replacing the complex subnucleonic structures by "effective" nucleons interacting through "effective" forces, the dimension of the nuclear many-body problem is overwhelming. Nuclei are exceedingly difficult to describe: they contain too many nucleons to allow for an exact treatment and far too few to disregard finite-size effects. Also, the time scale characteristic of collective nuclear modes is close to the single-nucleonic time scale. This means that many concepts and methods applied successfully to other many-body systems. such as solids and molecules, cannot be blindly adopted here.

\subsection{Nuclear forces}

The common theme for the whole field of nuclear structure is the problem of lorce: the one acting between two colliding nucleons, the one which produces exotic topologies in light nuclei, and that giving rise to the collective motion in heavy nuclei. The main challenges in understanding the nuclear force are shown in Fig. 1, in the context of the hadronic and nucleonic many-body problem.

The low-energy interaction between nucleons has complicated spin-isospin depenclence dictated by the hadron's substructure. One of the main challenges of nuclear science, indicated by the first bridge in Fig. 1, is the derivation of a nucleon-nucleon $(N V)$ interaction from the underlying quark-gluon dynamics of QCD. Experimentally, the $N N$ force can

*Supported by the U.S. Department of Energy through Contract No. DE-FG02-96ER40963 with the University of Tennessee. Oak Ridge National Laboratory is managed for the U.S. Department of Energy by Lockheed Martin Energy Research Corporation under Contract No. DE-AC O5-96OR22464. 


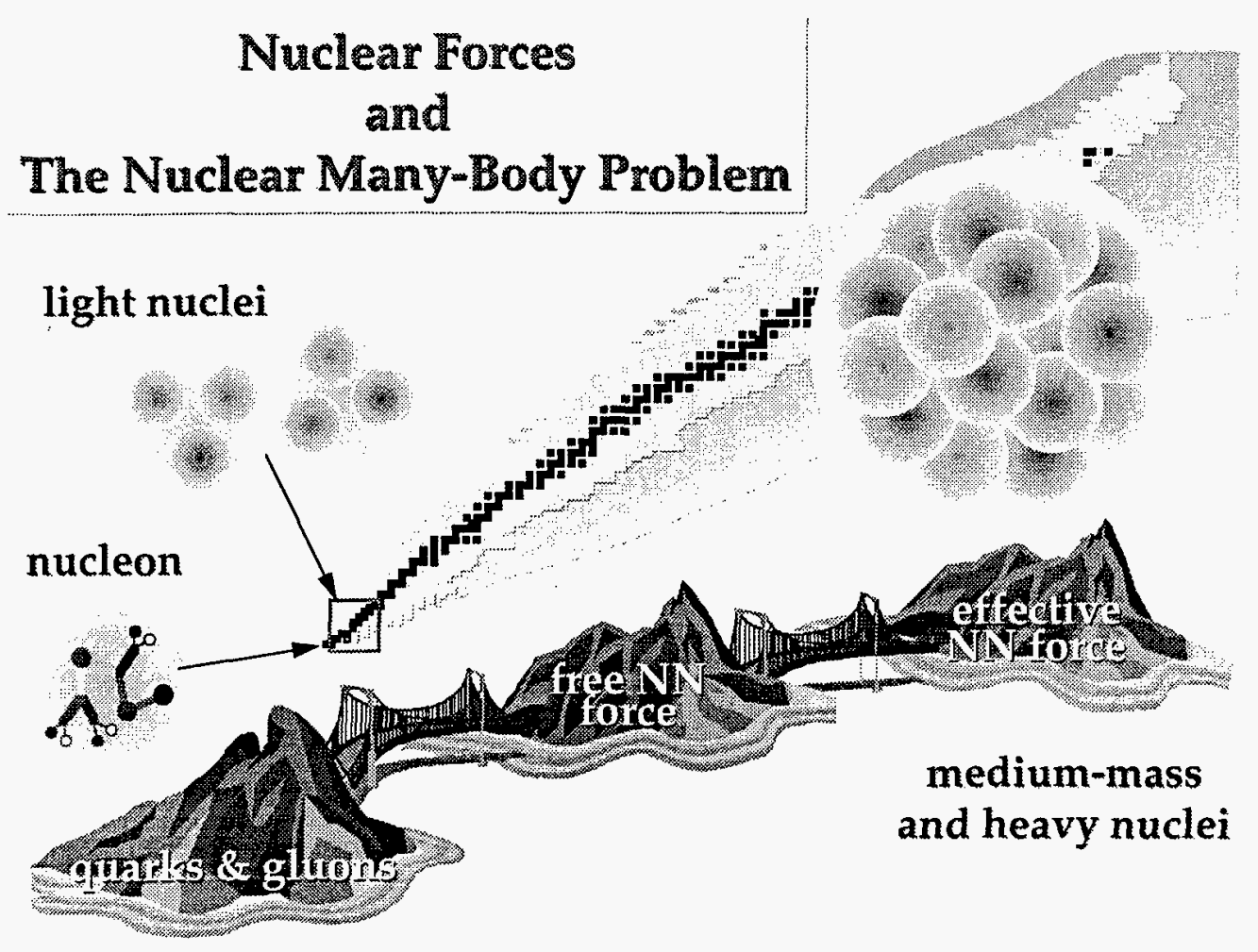

Figure 1. From hadrons to heavy nuclei: main challenges in understanding the nuclear force.

be studied by means of $N N$ scattering experiments. A large body of clata has been used to parameterize the low-energy $N N$ force. Examples for such parameterizations are the Bonn, Paris, Nijmegen, and Argonne potentials. While the long-range part of these free (but still effective!) $N N$ forces is well described by the one-pion exchange potential, their short-range behavior is not fully understood. Here the quark-gluon degrees of freedom must be considered explicitly.

While the very light nuclei can nowadays be described as $A$-body clusters bound by a free $N N$ force (including higher-order interactions, such as a three-body force), the conceptual framework of larger nuclei is still that of the nuclear shell model. Here, the basic assumption is that the nucleons are moving almost independently in a mean potential obtained by averaging out the interactions between a single nucleon and all remaining $A$ 1 protons and neutrons. The "effective" $N N$ interaction in the heavy nucleus used to determine the mean potential differs considerably from the free $N N$ force. In principle, it should be obtained by means of the complicated Brückner renormalization procedure which corrects the free $N N$ interaction for the effects due to the nuclear medium, and there has been some progress in this area (see Refs. [1.2] for recent developments). This challenging task is represented by the second bridge in Fig. 1. Many features of the effective interaction, such as short range; strong dependence on spins, isospins, and relative momenta of interacting nucleons; and the reduction of mass in the nuclear interior, have been extracted from experimental data. 
Figure 1 illustrates the intellectual connection between the hadronic many-body problem (quark-gluon description of a nucleon) and the nucleonic many-body problem (nucleus as a system of $Z$ protons and $N$ neutrons). The free $N N$ force can be viewed as a residual interaction of the underlying quark-gluon dynamics of QCD, similar to the intermolecular forces that stem from QED. Similarly, the effective $N N$ force in heavy nuclei can be derived from the effective free $N N$ interaction. It probably would be very naive to think of the behavior of a heavy nucleus directly in terms of the underlying quark-gluon dynamics, but - clearly - the understanding of the bridges in Fig. 1 will make this goal qualitatively possible.

\subsection{Theoretical developments}

The best $N N$ force parameterizations not only describe the two-body on-shell properties but have been used in few-body and many-body calculations. Probably the most advanced few-body calculations today are the quantum Monte Carlo calculations with the ArgonneUrbana interaction for nuclei with $A \leq 7$ [3]. The variational Monte Carlo calculations with a free $N N$ force have been carried out for relatively heavy systems such as ${ }^{16} \mathrm{O}[4]$.

What about the shell-model treatment of heavier nuclei? In the past, shell model calculations utilizing the concept of valence nucleons interacting in a restricted configuration space were limited to medium-mass nuclei owing to the rapid growth of the size of the model space. Today, this is still the case, although the conventional shell-model calculations employing realistic $N N$ interactions $[5,6]$ are becoming more and more efficient in handling large configuration spaces. The state-of-the-art shell-model studies of the $A=47$ and $49 f p$ nuclei in the full $0 \hbar \omega$ space [7] set the new standard in this area, although the future progress is strongly limited by present-day computer resources. Actually, it took two generations of hardware and software development to extend shell-model calculation:s from $A=44$ to $A=48$ ! (Conventional shell-model calculations for the $A=48$ nuclei involve model spaces with dimensions of several millions.) Hence, traditional shell-model calculations for heavy nuclei appear out of reach in the near future. The recently developed shell-model Monte Carlo method [8] overcomes the dimension barrier; it represents the interaction between valence nucleons by auxiliary fields. Applications of the Monte Carlo approach to the nuclear shell model have been remarkably successful in describing many structural properties of heavy nuclei such as ${ }^{64} \mathrm{Ge}[9]$ or ${ }^{124} \mathrm{Xe}[10]$. (Here, the dimension of the model space is of the order of $10^{13}$ !)

For nuclei close to the magic ones, the low-energy properties depend primarily on the behavior of a few valence nucleons. However, for nuclei with many valence particles, the concept of valence nucleons is less useful, and the valence and inner-shell nucleons have to be treated on an equal footing. Many properties of these heavy systems are well clescribed by means of self-consistent theories with the density-dependent effective $N N$ interaction. Here, the static mean-field ciescription, based on the Hartree-Fock (HF), Hartree-FockBogolyubov (HFB) method, or relativistic mean field (RMF) theory, provides a useful starting point. Thanks to developments in computational techniques, the approaches employing microscopic effective interactions are now widely used and - in terms of their predictive power - favorably compare with results of more phenomenological macroscopicmicroscopic models. Examples of recent large-scale self-consistent mean-field calculations include HF [11], HFB [12], and RMF [13] studies of ground-state nuclear properties. 


\subsection{Nuclear structure at the limits}

What are the frontiers of nuclear structure today? For light nuclei, one of such frontiers is physics at subfemtometer distances where the internal quark-gluon structures of nucleons overlap. For heavier nuclei, the frontiers are defined by the extremes of (i) the $N / Z$ ratio (Sec. 2), (ii) atomic charge and nuclear mass (Sec. 3), and (iii) angular momentum (Sec. 4). The tour to the limits is not only a quest for new and unexpected phenomena (sometimes dubbed as a "fishing expedition"); the new data are expected as well to bring qualitatively new information about the effective $N N$ interaction and hence about the fundamental properties of the nucleonic many-body system. By exploring exotic nuclei one can magnify certain terms of the Hamiltonian which are small in "normal" nuclei, thus difficult to test. The hope is that after probing these important interactions at the limits, we can later improve the description of normal nuclei (at ground states, close to the valley of beta stability, etc.).

\section{LIMITS OF EXTREME $N / Z$ RATIOS}

Physics of radioactive nuclear beams (RNB) is one of the main frontiers of nuclear science today. Experimentally, thanks to technological developments, we are on the verge of invading the territory of extreme $N / Z$ ratios in an unprecedented way [1+.15].

The nuclear landscape, the territory of the RNB physics, is shown in Fig. 2. Black squares indicate stable nuclei; there are less than 300 stable nuclei, or those long-lived, with half-lives comparable to or longer than the age of Earth. Some of the unstable nuclei can be found on Earth, some are man-made (actually, as many as $\sim 2.200$ nuclei have been produced in nuclear laboratories), and several thousand uuclei are the yetunexplored exotic species. Moving away from stable nuclei by adding either protons or neutrons, one finally reaches the particle drip lines where the nuclear binding ends. The nuclei beyond the drip lines are unbound to nucleon emission; that is, for those systems the strong interaction is unable to cluster $A$ nucleons as one nucleus. (Exceptions are neutron stars lying far far away from the neutron drip line. These giant nuclei. having masses of $\sim 1.4$ of the mass of the Sun and radii of $\sim 5$ miles, exist thanks to gravitation, an interaction which can safely be neglected in the description of "normal" nuclei!)

The uncharted regions of the $(N, Z)$ plane contain information that can answer many questions of fundamental importance for science: How many protons and neutrons can be clustered together by the strong interaction to form a bound nucleus? What are the properties of very short-lived exotic nuclei with an extreme neutron-to-proton ratio $N / Z$ ? What is the effective nucleon-nucleon interaction in the nucleus having a very large neutron excess? There are also related questions in the field of nuclear astrophysics. Since radioactive nuclei are produced in many astrophysical sites, knowledge of their properties is crucial for our understanding of the underlying processes [16].

Where lie the particle drip lines? As seen in Fig. 2, the proton drip line lies relatively close to the line of beta stability; hence it is easy to reach experimentally (actually, it has been delineated up to bismuth). However, since neutrons do not repel each other by the Coulomb force, many neutrons can be added to nuclei starting from the valley of stability: As a result, the "lever arm" separating the neutron drip line from the valley of stability is large and difficult to probe experimentally; except for the lightest nuclei. the bounds of 


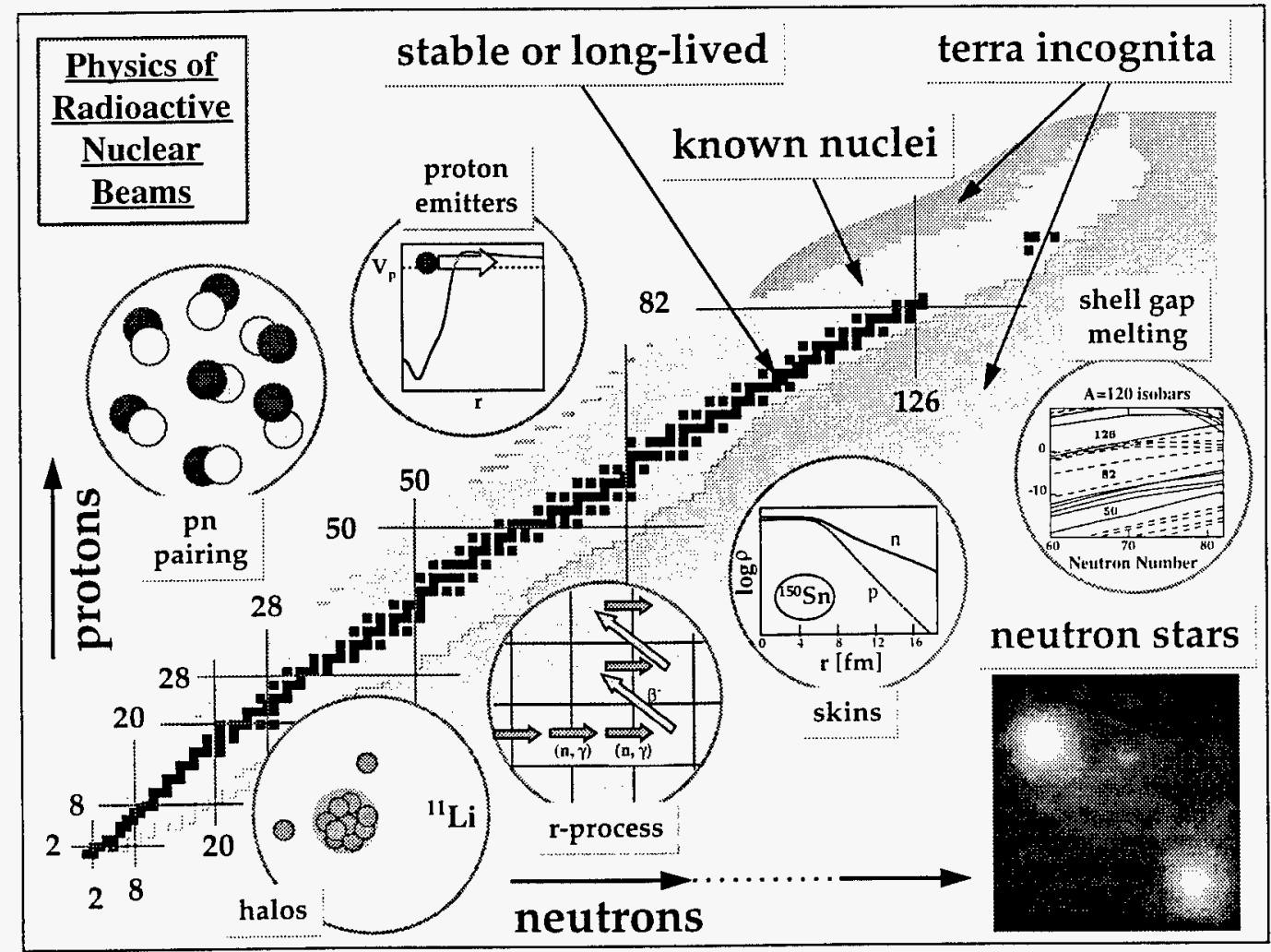

Figure 2. The nuclear landscape: territory of radioactive ion beam physics. Some of the important physics bullets are indicated schematically.

the neutron stability are not known.

Nuclear life at extreme $N / Z$ ratios is different from that around the stability line. The unique structural factor is the weak binding; hence the closeness to the particle continuum.

\subsection{Theoretical aspects of physics at extreme $N / Z$ ratios}

From a theoretical point of view, spectroscopy of exotic nuclei offers a unique test of those components of effective interactions that depend on the isospin degrees of freedom [17]. Effective interactions used to describe heavy nuclei are usually approximated by: means of density-dependent forces with parameters that are usually fitted to stable nuclei and to selected properties of the infinite nuclear matter. Hence, it is by no means obrious that the isotopic trends far from stability, predicted by commonly used interactions (such as Skyrme or Gogny forces), are correct. In the models aiming at such an extrapolation. the important questions asked are: What is the $N / Z$ behavior of the two-body central force and the one-body spin-orbit force $[18-20]$ ? Does the spin-orbit splitting strongly vary with $N / Z[21]$ ? What is the form of the pairing interaction in weakly bound nuclei $[22,23]$ ? What is the importance of the effective mass (i.e., the non-locality of the force) for isotopic trends [24]? What is the role of the medium effects (renomalization) and 
of the core polarization in the nuclear exterior (halo or skin region) where the nucleonic density is small [25]?

Exotic nuclei are wonderful laboratories to study superfluid correlations. Here, the main questions pertaining to the problem of pairing force are: What is the microscopic origin of the pairing interaction $[26,27]$ ? What is the role of finite range and the importance of density dependence $[22,28]$ ? How can properties of the pairing force be tested? These questions are of considerable importance not only for nuclear physics but also for nuclear astrophysics and cosmology [29-32]. For instance, a better understanding of the density dependence of the nuclear pairing interaction is important for theories of superfluidity in neutron stars. It is impossible at present to deduce the magnitude of the pairing gaps in neutron stars with sufficient accuracy. Indeed, calculations of ${ }^{1} S_{0}$ pairing gaps in pure neutron matter, or symmetric nuclear matter based on free $N N$ interactions, suggest a strong dependence on the force used; in general, the singlet- $S$ pairing is very small at the saturation point. On the other hand, nuclear matter calculations with effective finite-range interactions yield rather large values of the pairing gap at saturation!

As mentioned above, the main theoretical challenge is the correct treatment of the particle continuum. For weakly bound nuclei, the Fermi energy lies very close to zero, and the decay channels must be taken into account explicitly. As a result, many cherished approaches of nuclear theory such as the conventional shell model, the pairing theory, or the macroscopic-microscopic approach must be modified. But there is also a splendid opportunity: the explicit coupling between bound states and continuum, and the presence of low-lying scattering states invite strong interplay and cross-fertilization between nuclear structure and reaction theory.

\subsection{Physics of large neutron excess}

The intense current interest in experimental exploration of the neutron drip line region is driven not only by the substantial uncertainties in theoretical predictions of its location, but also by the expectation that qualitatively new features of nuclear structure will be discovered in this exotic territory. What makes neutron-rich nuclei so unusual? Firstly, they have very large sizes - as implied by their weak binding. Secondly, they are very diffused; their properties are greatly dominated by surface effects. Thirdly, they are very superfluid; the close-lying particle continuum provides a giant reservoir for scattered neutron Cooper pairs [33,22]. So, roughly speaking, they are large fuzzy superfluids.

Extreme cases are halo nuclei - loosely bound few-body systems with about thrice more neutrons than protons [14,34.35]. The halo region is a zone of weak binding in which quantum effects play a critical role in distributing nuclear clensity in regions not classically allowed. In the heavier, neutron-rich nuclei, where the concept of mean field is better applicable, the separation into a "core" and "valence nucleons" seems less justified. However, also in these nuclei the weak neutron binding implies the existence of the neutron skin (i.e., a dramatic excess of neutrons at large distances). In the skin region of hearier, very neutron-rich nuclei, one may find the opportunity to study in the laboratory nearly pure neutron matter at densities much less than the normal nuclear density. In addition, the existence of a neutron skin should lead to new collective vibration modes in such nuclei. in which. for example, the neutron shin may oscillate out of phase with a wellbound proton-neutron core. 


\subsection{Nuclear shell structure far from stability}

The structure of nuclei is expected to change significantly as the limit of nuclear stability is approached in neutron excess. Due to the systematic variation in the spatial distribution of nucleonic densities and the increased importance of the pairing field, the average nucleonic potential is modified [36]. This results in a new shell structure characterized by a more uniform distribution of normal-parity orbits and the unique-parity intruder orbit which reverts towards its parent shell.

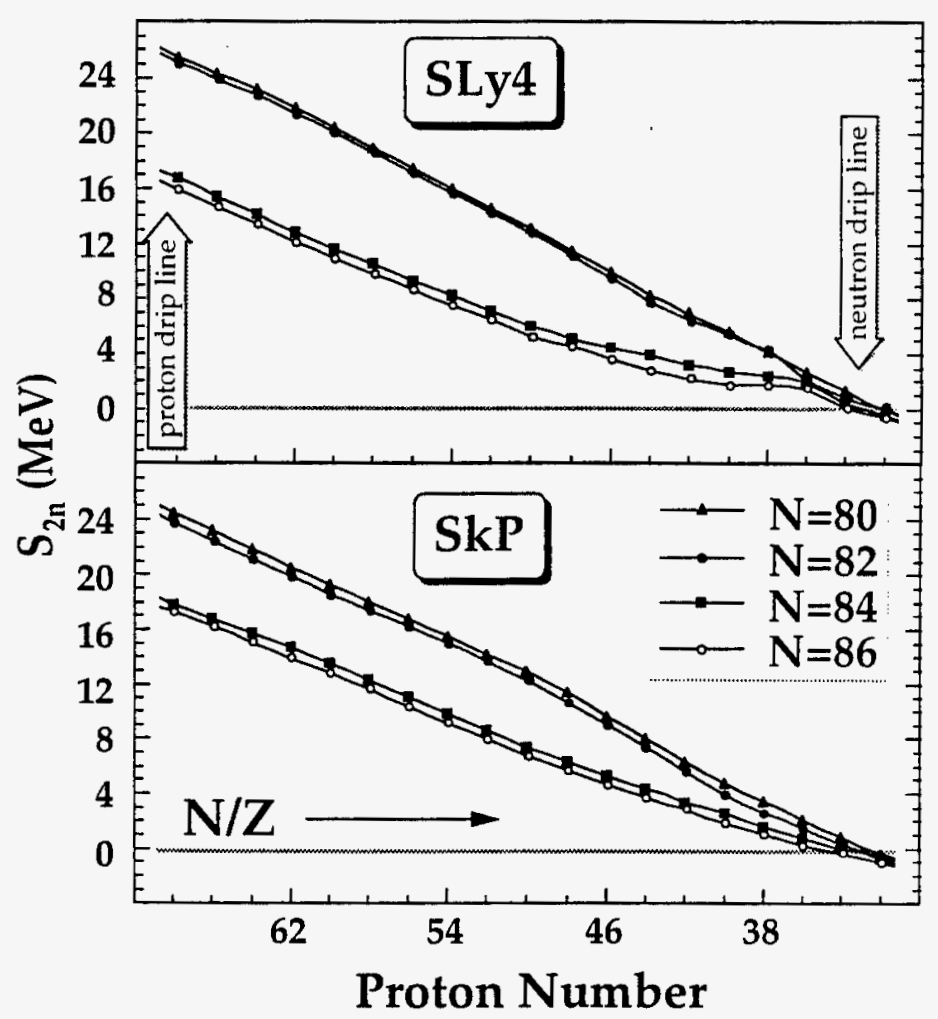

Figure 3. Two-neutron separation energies for the $N=80,82,84$, and 86 spherical ermeven isotones calculated in the $\mathrm{HFB}+\mathrm{SkP}$ and $\mathrm{HFB}+\mathrm{SLy} 4^{\circ}$ models as functions of the proton number. The arrows indicate the proximity of neutron and proton drip lines for small and large proton numbers, respectively. (From Ref. [17].)

The quenching of shell effects manifests itself in the behavior of two-neutron separation energies $S_{2 n}$. This is illustrated in Fig. 3. which displays the two-neutron separation energies for the $N=80,82,84$, and 86 spherical even-even isotones calculated in the HFB model with the SkP and SLy4 effective interactions. The large $N=\$ 2$ magic gap, clearly seen in the nuclei close to the stability valley and to the proton drip line, gradually closes down when approaching the neutron drip line. As discussed in Refs. [16.37]. this has important consequences for the r-process and the stellar nucleosynthesis.

It is to be noted that the experimentally observed collapse of magic gaps seen in some neutron-rich light nuclei, e.g.: around ${ }^{32} \mathrm{Mg}_{20}$ and ${ }^{44} \mathrm{~S}_{28}$ (see Ref. [38]), is conventionally explained in terms of the shape transition to the deformed intruder configuration. In both 
cases, HF calculations predict the shape transition $[39,40]$ due to the crossing of spherical configuration by intruder states. To what extent the appearance of a low-lying deformed configuration is influenced by the shell-melting effect still remains an open and exciting problem.

\subsection{Physics of proton-rich nuclei: nuclear life at and beyond the drip line}

On the proton-rich side of the valley of stability, physics is different than in nuclei with a large neutron excess. Because of the Coulomb barrier which tends to localize the proton density in the nuclear interior, nuclei beyond the proton drip line are quasibound with respect to proton emission. However, in spite of the stabilizing effect of the Coulomb barrier, the effects associated with the weak binding are also present in proton drip line nuclei. They are not as dramatic as on the other side of the stability valley, but nevertheless important [17].

The doubly magic $N=Z=50$ nucleus ${ }^{100} \mathrm{Sn}$ is a paradigm of RNB physics on the protonrich side. Although it was found experimentally three years ago [41.+2]. it took more than two years to roughly determine its mass [43], and it will probably take several vears to find its first excited state. Actually, the question. "what is this state?", constitutes an unresolved problem which is a challenge for theoretical predictions.

A unique aspect of proton-rich nuclei with $N=Z$ is that neutrons and protons occupy the same shell-model orbitals. Consequently, due to the large spatial overlaps between neutron and proton single-particle wave functions, the proton-rich $N=Z$ nuclei are expected to exhibit unique manifestations of proton-neutron ( $p n$ ) pairing [44.45] carried by isotropic $(S=0, T=1)$ and anisotropic, doughnut-shaped $[46](S=1, T=0)$ proton-neutron ('ooper pairs.

At present, it is not clear what the specific experimental fingerprints of the $p n$ pairing are, whether the $p n$ correlations are strong enough to form a static pair condensate, and what are their main building blocks [47]. So far, the strongest evidence for enhanced $p n$ correlations around the $N=Z$ line comes from the measured binding energies. An additional binding (the so-called Wigner energy) found in these nuclei manifests itself as a spike in the isobaric mass parabola as a function of $T_{z}=\frac{1}{2}(N-Z)$ [48.49]. Recent calculations [50] have revealed the rather complex mechanism responsible for the nuclear binding around the $N=Z$ line. In particular, it has been found that the Wigner term cannot be solely explained in terms of correlations between the proton-neutron $J=1$, $T=0$ (deuteron-like) pairs. The $p n$ correlations are also expected to play a role in beta decay [51,52], deuteron transfer reactions [5.3], structure of high spins [45,54.55], and also in nuclear matter $[31,56]$.

Proton-rich nuclei also offer the unique opportunity to study life beyond the drip line. Although the protons in this region are strictly not bound to the nuclear core. nonetheless their escape is impeded by the Coulomb force which is not present for neutrons. Quantum barrier tunneling allows these nuclei to decay by proton emission. but with lifetimes ranging from microseconds to a few seconds - long enough that one can measure their spectroscopic properties [57,58]. Experimentally, a number of proton emitters have now been discovered in the mass regions $A \sim 110,150$, and 170 [59]. However, it is anticipated that new regions of proton-unstable nuclei will be explored in the near future using RNB's.

Proton radioactivity is an excellent example of the elementary three-dimensional tun- 


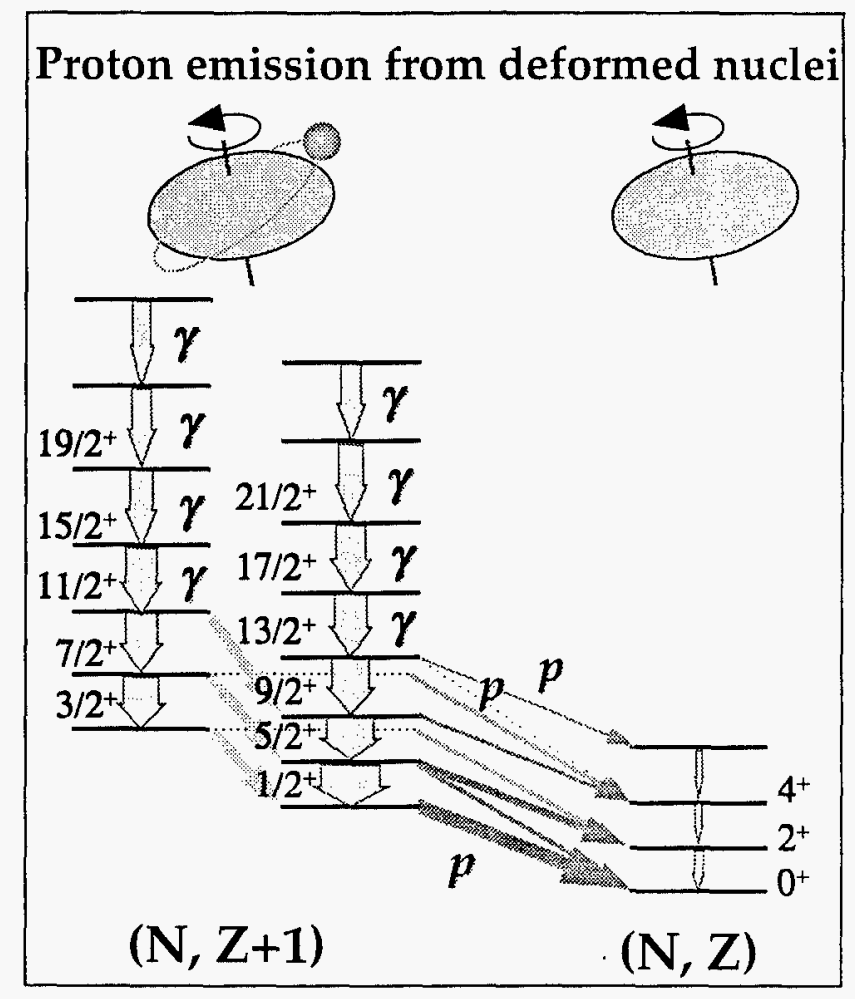

Figure 4. Competition between gamma and proton decays in a deformed proton emitter.

neling. Experimental and theoretical investigations of proton emitters (or theoretically. predicted ground-state di-proton emitters) will open up a wealth of exciting physics associated with the residual interaction coupling between bound states and extremely narrow resonances in the region of very low density of single-particle levels. In general. proton emission half-lives depend mainly on the proton separation energy and orbital angular momentum. but rather weakly on the details of the intrinsic structure of proton emitters. e.g., on the parameters of the proton-core potential. This suggests that the lifetimes of deformed proton emitters will provide direct information on the angular momentum content of the associated Nilsson state, and hence on the nuclear shape. Figure $t$ shows. schematically, a possible scenario of proton-gamma competition in a deformed proton emitter. Of course, the energy window for such a process is expected to be fairly narrow. I slighty different phenomenon, gamma-delayed proton emission, has been recent ly observed in the nucleus ${ }^{58} \mathrm{Cu}[60]$. Here the strongly deformed excited intruder band gamma-decays to the lower-lying states. but its proton-unstable band head directly feeds into the excited state in ${ }^{57} \mathrm{Ni}$.

\section{LIMITS OF MASS AND CHARGE}

The dawn of the nuclear age dates back to one hundred years ago when Henri Becquerel and the Curies discovered the phenomenon of radioactivity. This laid the foundation for nuclear chemistry, and changed the face of science. technology: and merlicine. The modern 
periodic table, as of 1997 , ends with the 112 th element now known. But the search is still going on; we may be due for a major expansion at the upper end of the periodic table [61].

The discovery of the properties of a brand new set of chemical elements can answer questions of fundamental importance for science: What is the maximum charge and mass that an atom and nucleus may attain? Is there a limit to the number of protons that can be contained in one nucleus? What are the closed electron shells of the atom on the one hand, and what are the proton and neutron magic numbers of the new elements, on the other hand?

Due to the strong Coulomb repulsion between protons, the nuclear liquid drop would fission immediately for $Z>104$. However, the quantal shell effects allow very heavy elements to exist. Since the first theoretical predictions of the region of superheavy elements in the mid-sixties, and Herculean experimental efforts in a number of the world's leading nuclear laboratories, it is only now that the new heavy elements with $Z \geq 110$ have been produced [62-65]. These isotopes decay predominantly by groups of a-chains. The experimental chain of $\alpha$ decays that identifies the heaviest known element with $A=2 \pi 3$ and $Z=112$ is shown in Fig. 5 .

All the heaviest elements found recently are believed to be well deformed. Indeed, the measured $\alpha$-decay energies, along with the complementary syntheses of new neutronrich isotopes of elements $Z=106$ and $Z=108$, have furnished confirmation of the special stability of the deformed shell at $N=162$ [66-68]. Still heavier and more neutron-rich elements are expected to be spherical and even more strongly stabilized by shell effects: they form the long predicted region of the superheavy elements (SIIE). The discorery of these elements would provide a critical test of not only nuclear morlels. but also of relativistic quantum chemistry. According to relativistic calculations. the velocity of the inner-shell electrons is predicted to approach the velocity of light as the atomic number of a nucleus grows up to 173. The resulting relativistic effects canse deviations from the periodicity of chemical properties that characterize the periodic table for lighter elements.

Where is the center of superheavy shell stability expected to fall? For the neutrons, most calculations predict an increased stability at $N=184$. However, theorists are not unanimous with regard to the position of the magic proton number. Indeed. in the region of the SHEs, the Coulomb interaction cannot be treated perturbatively; and the self-consistent coupling between nuclear and electromagnetic parts of the Hamiltonian becomes essential. While earlier macroscopic-microscopic calculations yielded the value $Z=114[69-71]$, modern models favor $Z=126$ or $Z=120$ [72.73]. According to recent HF calculations of Ref. [72], the valley of shell stability extends from the deformed region around $Z=108, N=162$ towards the spherical doubly magic nucleus $A=310, Z=126$ which is actually predicted to decay via $\alpha-, \beta$-, $p$-radioactivity, and fission!

The contour map of shell energy calculated in the HF+Sly 7 model of Ref. [72] is displayed in Fig. 5. The calculation clearly shows the presence of strong shell stability around the "doubly magic" nucleus ${ }^{310} 126$. This result markedly differs from the most macroscopic-microscopic approaches where the island of shell stability is concentrated around $Z=114$. The HF prediction is consistent with the recent results of the experimental systematics of $B(E 2)$ rates [7t], according to which $Z=126$ is presumably the dominant proton shell closure when the global properties of nuclei in the trans-actinide 


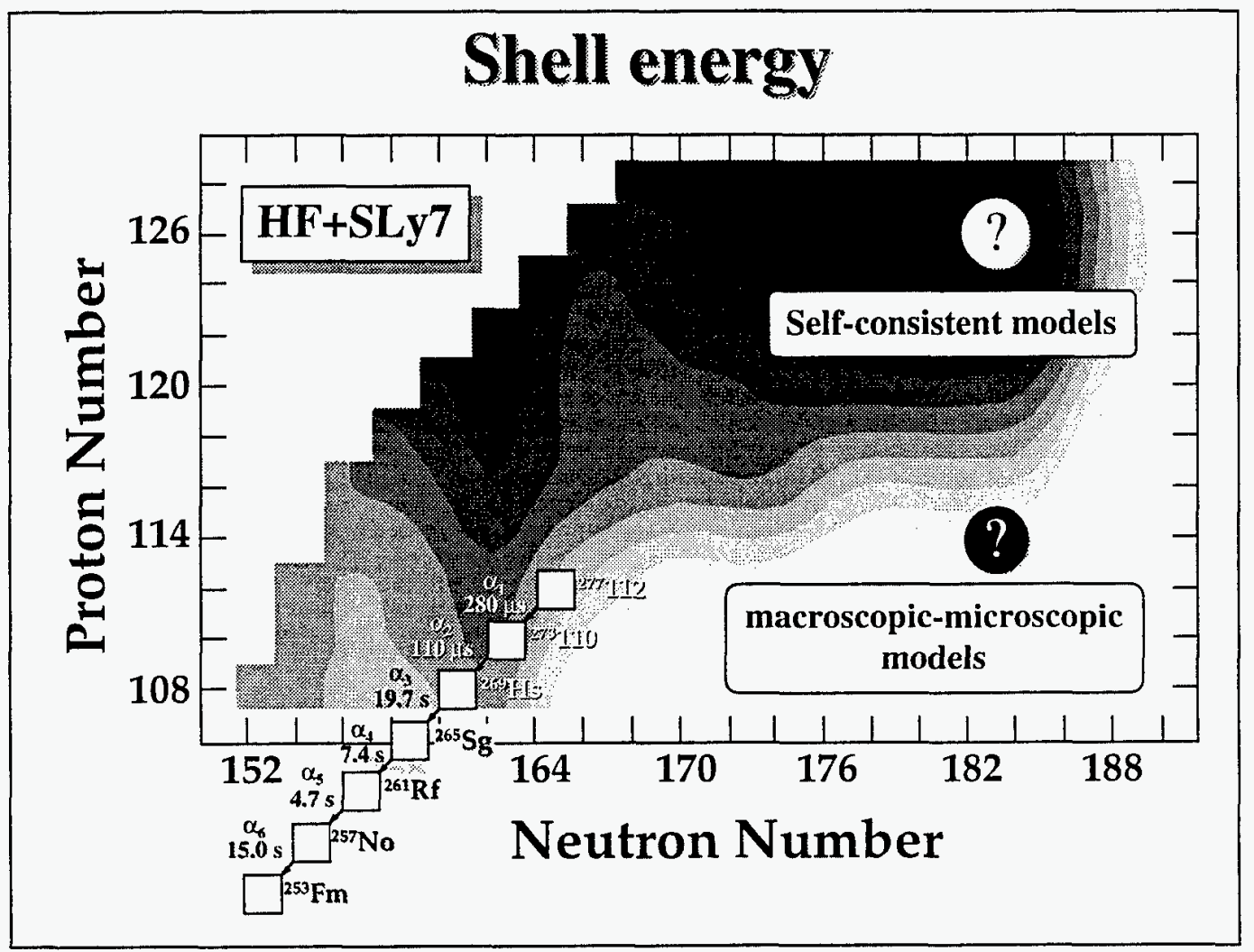

Figure 5. Contour map of shell energy for the SHE obtained with the self-consistent HF +SLy 7 model of Ref. [72]. The experimental chain of $\alpha$-particle decays that identifies the currently heaviest known element with $A=273$ and $Z=112$ [63] is indicated.

region are parameterized in terms of numbers of valence particles.

\section{LIMITS OF ANGULAR MOMENTUM}

Considering that the atomic nucleus is, in a good approximation, an aggregation of strongly interacting nucleons (or pairs of nucleons) moving solo in all clirections. the very existence of nuclear collective motions such as rotations or vibrations, with all particles moving in unison, is rather astonishing. In molecular physics, for example, the fast electronic motion is strongly coupled to the equilibrium position of slowly moving ions. Consequently, the adiabatic assumption is justified due to different time scales. In atomic nuclei, the total $A$-body wave function cannot, in general, be expressed in terms of slow and fast components. This is because (i) the collective nuclear coordinates are auxiliary variables which depend, in a complex way, on fast nucleonic degrees of freedom and (ii) the nuclear residual interactions are large.

How good is the time separation between single-particle and collective nuclear motion? The typical single-particle period (i.e.. the average time it takes a neutron or a proton to go across the nucleus), $T_{\text {s.p. }}=4 R / v_{\mathrm{F}}$ [where $R$ is the nuclear radius and $\iota^{\prime} \mathrm{F}$ is the Fermi 
velocity $(\sim 0.29 \mathrm{c})]$, is approximately $3 \cdot 10^{-22} \mathrm{sec}$. The typical period of nuclear rotation $\left(T_{\text {rot }} \approx 10^{-21} \mathrm{sec}\right)$ is only $\sim 30$ times greater than $T_{\text {s.p. }}$, and for nuclear vibrations the period of oscillations is only slightly greater than the single-particle period. It is truly amazing that these relatively small differences in time scales seem to be sufficient to create rotating or vibrating potentials, common for all nucleons! Indeed, as remarked earlier in Ref. [75]: "In contrast with the molecular case, there are here no heavy particles to provide the necessary rigidity of the structure. However, nuclear matter appears to have some of the properties of coherent matter which makes it capable of types of motion for which the effective mass is large, as compared with the mass of a single nucleon."

One of the outstanding challenges in nuclear structure is to understand the mechanism governing the nature of nuclear collective excitations. By studying nuclear rotations and vibrations, one is probing the details of the nuclear force in the strongly interacting medium.

\subsection{Territory of high-spin physics}

Figure 6 displays, schematically, the territory of nuclear high-spin science. The preferred gamma-ray pathways observed in the de-excitation process can often be associated with specific nuclear deformations. Some spectacular examples of intrinsic shapes are illustrated in Fig. 6: pear-like shapes characteristic of parity breaking in the intrinsic system, superdeformed (SD) and hyperdeformed (HD) shapes corresponding to huge elongations of nuclear density, magnetic deformations represented by shears bands, or static pairing deformations that give rise to the nuclear Josephson effect and diabolic pair transfer.

One of the most exciting recent discoveries is the link between SD bands and previously known less-deformed states in several nuclei around ${ }^{192} \mathrm{Hg}$ [76.7T]. This direct connection makes it possible to determine the absolute binding energy of the nuclear SD state and hence the magnitude of shell effects (including proton and neutron separation energies) in the SD configuration. This information is critical for the further discrimination between various effective interactions, previously applied successfully to high-spin states [78]. Another important achievement has been the identification of single-particle excitations in the rotating SD well through their electric and magnetic properties. This has been accomplished by measurements of intraband magnetic transitions $[79,80]$ and relative electric quadrupole moments [81-83]. Discussed below are some of the bullets shown in Fig. 6.

\subsection{Superdeformation: Spectroscopy at a keV Scale}

The observation of SD states constitutes an important confirmation of the shell structure of the nucleus. Quantum-mechanically, the remarkable stability of SD states can be attributed to strong shell effects that are present in the average nuclear potential at very elongated shapes [84-86]. For the oscillator potential, this happens when the frequency ratio is $2: 1$. (For more realistic average potentials, strong shell effects appear even at lower deformations.) The structure of single-particle states around the Fermi level in SD nuclei is significantly different from the pattern at normal deformations. Indeed. the SD shells consist of states originating from spherical shells having different principal quantum numbers, and hence having very different spatial character. This unusual situation produces new effects in nuclear structure.

Superdeformed states have been discovered in several mass regions. These are fission 


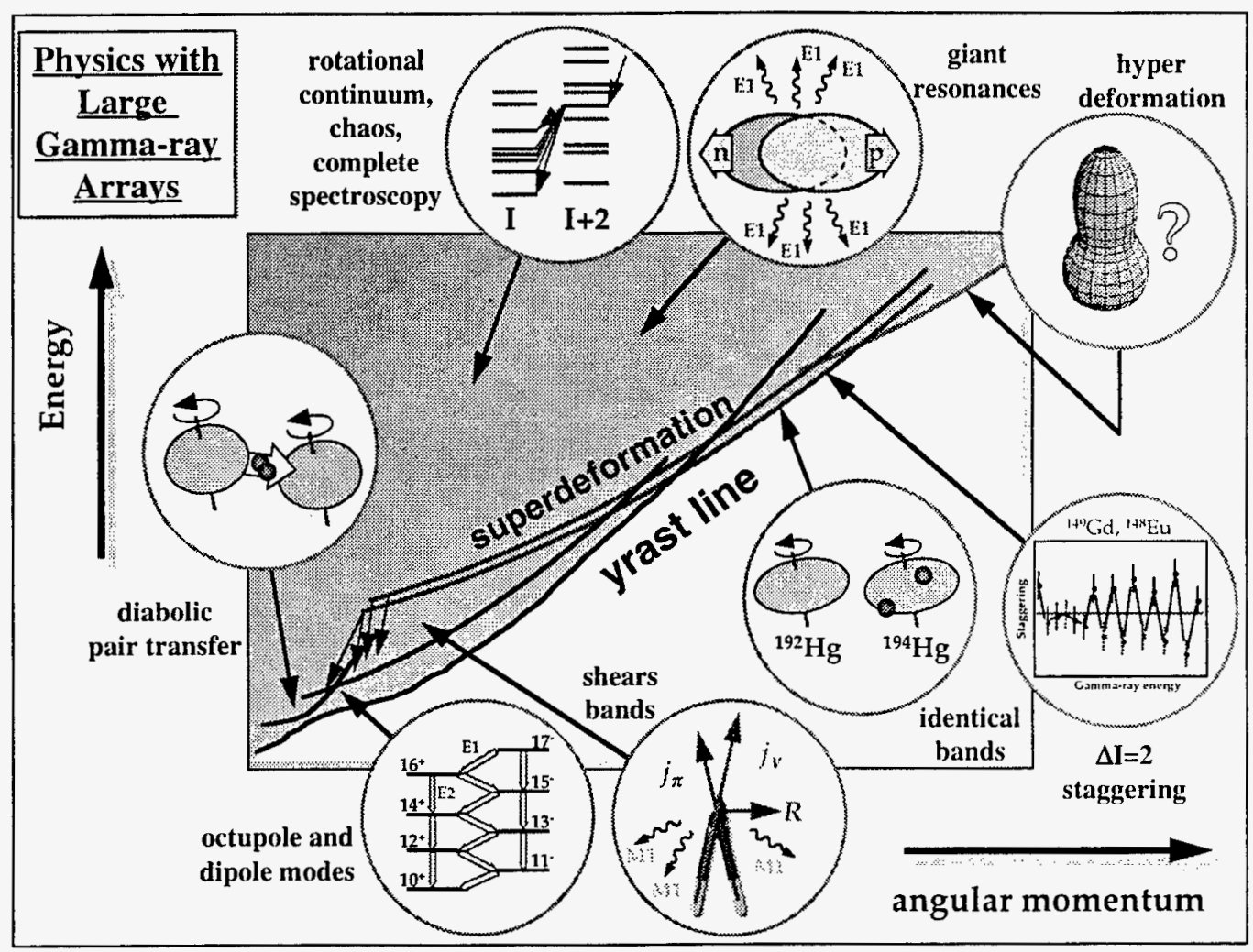

Figure 6. Territory of nuclear high spins. Some of the important physics bullets are indicated schematically.

[90]; and "molecular" (cluster) configurations in light nuclei [91]. Intrinsic configurations of SD states are well characterized by the intruder orbitals carrying large principal oscillator numbers $[92,93]$. Because of their large intrinsic angular moment a and cuadrupole moments, these orbitals strongly respond to the Coriolis interaction and to the cleformed average field.

The increased precision of experimental tools of gamma-ray spectroscopy has allowed us to look much more closely at very weak effects which are, energetically, at an $\mathrm{eV}$ or a $\mathrm{keV}$ scale. Among the most startling new phenomena is that of identical bands. i.e., the observation of sequences of ten or more identical gamma rays associated with rotational bands in different nuclei [94]. Another recent discovery in SD nuclei is the observation of very small (tens of eV!), but systematic, shifts in the energy levels of certain bands ( $\Delta I=2$ staggering) $[95,96]$.

A satisfactory explanation of both phenomena is still lacking [94] (see also recent Refs. [97.98]). It is rather clear that the puzzle of identical bands is ultimately related to the questions normally addressed in the context of large-amplitude collective motion, such as: What are the "strong" quantum numbers that stabilize collective rotation? Indeed, recent studies of moments of inertia and quadrupole moments in SD bands around ${ }^{152} \mathrm{Dy}$ [99] show that nuclear systems at very large deformations and high angular momenta are the best examples of an almost-undisturbed single-particle motion (i.e.. extreme one-body picture) [100.101]! 


\subsection{Magnetic rotation}

In nearly spherical nuclei, sequences of gamma rays were observed reminiscent of collective rotational bands, but consisting of unusually strong magnetic dipole transitions $[102,103]$. The basic mechanism leading to the unusually large magnetic collectivity is explained theoretically [104] in terms of a gradual alignment of valence protons and neutrons along the axis of the total spin (shears mechanism). Since quadrupole deformations of these bands are extremely small, the rotational-like pattern observed cannot be attributed to the rotation of the electric charge distribution. It is believed that the collective properties of shears bands are linked to magnetic-type static intrinsic deformations. However, the exact nature of the intrinsic magnetic fields responsible for this magnetic rotation is not fully understood at present.

\subsection{Complete spectroscopy and quasicontinuum: from chaos to order}

Gamma-ray spectroscopy with the new generation of detector systems offers a unique possibility to probe quantum chaos, roughly defined as a regime where the quantum numbers that may be used to characterize low-lying (cold) states of a many-body system are gone. Today, the important issues under study in the so-called "complete spectroscopy" experiments are: At what energy does chaos set in? What are the unique fingerprints of the transition from regular to chaotic motion? The signatures for the onset of chaos can be observed not only in the distribution of energy levels but also in the properties of electromagnetic transition intensities.

There are many splendid examples of an interplay between chaos and order in nuclear spectroscopy. At low excitation energies (low temperatures), there exist intrinsic quantum numbers that can well isolate and characterize nuclear configurations. At high excitation energies, because of a very large level density and dramatic configuration mixing. the concept of quantum numbers virtually breaks down. However. even at high excitation energies there still survive well-ordered states, dubbed "symmetry scars", that can be beautifully described by stable regular mean fields with well-defined quantum numbers: superdeformed states, high- $K$ isomers, coexisting structures, and analog states. One of the most important tasks for experiment and theory is to identify such islands of stability, and to understand the underlying quantum numbers, that is, to find order in chaos.

\subsection{Theoretical developments}

Unexpected experimental data from large arrays have generated a great deal of theoretical interest and activity. As a result, many new insights have been gained regarding the behavior of atomic nuclei at high spins.

The new-generation high-spin data tell us a lot about effective $N N$ interaction. and about pairing force in particular. For instance, self-consistent HF studies of moments of inertia of SD bands indicate the importance of higher-order pairing interactions such as the rotation-induced $K^{\pi}=1^{+}$pairing forces $[105,106]$ and the density-dependent pairing forces [10 -109$]$. Interestingly, the question of the density dependence of pairing interaction is also of great interest for physics of nuclear radii, deep hole states. and properties of drip line nuclei [22]. That is, this important problem has also surfaced in a different corner of nuclear structure.

In the microscopic theory of a rotating nucleus, the arerage nucleonic field is obtained self-consistently from the nucleonic density. Consequently. in the presence of a large 
angular momentum, the intrinsic density is strongly polarized, i.e., the nucleus shows phenomena and behaviors characteristic of condensed matter in the magnetic field: ferromagnetism, Meissner effect, Josephson effect. The nuclear magnetism is caused by the time-odd components in the average potential [110-112]. The understanding of the structure of these terms will rely mostly on the expected increase of information on high-spin properties and on magnetic properties. Here, a good example is recent calculations based on a RMF theory; as a consequence of broken time-reversal invariance, the spatial contributions of the vector meson fields have a strong influence on the moments of inertia [113].

\section{SUMMARY}

The main objective of this brief review was to discuss various facets of nuclear structure "at the limits". The list of topics covered is by no means complete due to time and space constraints. There is very little doubt that an experimental excursion into nuclear exotiru will offer many excellent physics opportunities. We are only beginning to explore many unusual aspects of the nuclear many-body problem offered by systems with extreme $N / Z$ ratios, extreme angular momenta, and extreme nuclear charges and masses.

\section{REFERENCES}

1. P. Navratil and B.R. Barrett, Phys. Rev. C54 (1996) 2986.

2. M. Hjorth-Jensen, T.T.S. Kuo and E.Osnes, Phys. Rep. 261 (199:5) 12.5.

3. B.S. Pudliner et al., nucl-th/9705009, to be published in Phys. Rev. (.

4. S.C. Pieper, R.B. Wiringa, and V.R. Pandharipande, Phys. Rev. C46 (1992) $17+1$.

5. F. Andreozzi et al., Phys. Rev. C54 (1996) 1636.

6. P. Navratil, M.Thoresen, and B.R. Barrett, Phys. Rev. C55 (1997) R573.

7. G. Martinez-Pinedo, A.P. Zuker, A. Poves, and E. Caurier, Phys. Rev. C55 (1997) 187.

8. S.E. Koonin, D.J. Dean, and K. Langanke, Phys. Rep. 278 (1997) 1.

9. M. Honma, T. Mizusaki, and T. Otsuka, Phys. Rev. Lett. 77 (1996) 3315.

10. Y. Alhassid, G.F. Bertsch, D.J. Dean, and S.E.Koonin, Phys. Rev. Lett. 77 (1996() 1444.

11. N. Tajima, S. Takahara, and N. Onishi, Nucl. Phys. A603 (1996) 23.

12. J. Dobaczewski, W. Nazarewicz, and T.R. Werner, Z. Phys. A354 (1996) 27.

13. D. Hirata et al., Nucl. Phys. A616 (1997) 438c.

14. P.G. Hansen, this volume.

15. K. Rykaczewski, this volume.

16. K.-L. Kratz, this volume.

17. J. Dobaczewski and W. Nazarewicz, Phil. Transactions, in press. 1997; nuclth/9707049.

18. J.M. Pearson and M. Farine, Phys. Rev. C50 (1994) 185.

19. P.-G. Reinhard and H. Flocard, Nucl. Phys. A584 (1995) 467 .

20. M. Onsi et al., Phys. Rev. C55 (1997) 3166.

21. B.S. Pudliner et al., Phys. Rev. Lett. 76 (1996) 2416.

22. J. Dobaczewski et al., Phys. Rev. C53 (1996) 2809. 
23. S.A. Fayans and D. Zawischa, Phys. Lett. 383B (1996) 19.

24. J. Dobaczewski, W. Nazarewicz and T.R. Werner, Physica Scripta T56 (1995) 15.

25. T.T. Kuo, F. Krmpotić, and Y. Tzeng, Phys. Rev. Lett. 78 (1997) 2708.

26. D.S. Delion, M. Baldo, and U. Lombardo, Nucl. Phys. A593 (1995) 151.

27. H. Kucharek and P. Ping, Z. Phys. A339 (1991) 23.

28. S.A. Fayans, S.V. Tolokonnikov, E.L. Trykov, and D. Zawischa. Phys. Lett. 338B (1994) 1.

29. C.J. Pethick and D.G. Ravenhall, Annu. Rev. Nucl. Part. Sci. 45 (1995) 429.

30. C.J. Pethick, D.G. Ravenhall, and C.P. Lorenz, Nucl. Phy̌s. A584 (1995) 675.

31. B.E. Vonderfecht et al., Phys. Lett. B253 (1991) 1.

32. F. De Blasio and G. Lazzari, Nuovo Cimento 107A (1994) 1.549.

33. J. Dobaczewski, H. Flocard and J. Treiner, Nucl. Phys. A422 (1984) 103.

34. J. Tostevin, this volume.

35. A.S. Jensen, this volume.

36. J. Dobaczewski, I. Hamamoto, W. Nazarewicz and J.A. Sheikh. Phı̌s. Rev. Lett. 72 (1994) 981.

37. B. Pfeiffer, K.-L. Kratz and F.-K. Thielemann, Z. Phys. A357 (1997) 235).

38. T. Glasmacher, this volume.

39. X. Campi, H. Flocard, A. K. Kerman, and S. Koonin, Nucl. Phys. A251 (1975) 193.

40. T.R. Werner et al., Phys. Lett. B333 (1994) 303.

41. R. Schneider et al., Z. Phys. A 348 (1994) 241.

42. M. Lewitowicz et al., Phys. Lett. B332 (1994) 20.

43. M. Chartier et al., Phys. Rev. Lett. 77 (1996) 2400.

44. A. Goswami, Nucl. Phys. 60 (1964) 228.

45. A.L. Goodman, Adv. Nucl. Phys. 11 (1979) 263.

46. J.L. Forest et al., Phys. Rev. C54 (1996) 646.

47. W. Nazarewicz and S. Pittel, http://aps.org/BAPSAPR97/rpr/laye8.html.

48. N. Zeldes, in: Handbook of Nuclear Properties, ed. by D. Poenaru and W. Greiner. Clarendon Press, Oxford, 1996, p. 13.

49. D.S. Brenner et al., Phys. Lett. 243B (1990) 1.

50. W. Satula et al., Phys. Lett. 407B (1997) 103.

51. J. Schwieger, F. Simkovic, and A. Faessler, Nucl. Phys. A600 (1996) 179.

52. J. Engel et al., Phys. Rev. C55 (1997) 1781.

53. P. Fröbrich, Phys. Lett. 37B (1971) 338.

54. J. Kvasil, A.K. Jain, and R.K. Sheline, Czech. J. Phys. 40 (1990) 278.

55. W. Satula and R. Wyss, Phys. Lett. B393 (1997) 1.

56. T. Alm, G. Röpke, and M. Schmidt, Z. Phys. 337 (1990) 35.5.

57. C.N. Davids, this volume.

58. S. Âberg, P.B. Semmes, and W. Nazarewic: Phys. Rev. C. October 1997.

59. S. Hofmann, in: Nuclear Decay Modes, ed. by D.N. Poenaru and W: Greiner, (IOP. Bristol, 1996); Preprint GSI-93-04, 1993.

60. D. Rudolph et al., this volume.

61. G. Münzenberg, this volume.

62. S. Hofmann et al., Z. Phys. A350 (1995) 277; ibid.. p. 281.

63. S. Hofmann et al. Z. Phys. A354 (1996) 229. 
64. A. Ghiorso et al., Nucl. Phys. A583 (1995) 861c.

65. Y.T. Oganessian, Nucl. Phys. A583 (1995) 823c.

66. S. Ćwiok, V.V. Pashkevich, J. Dudek and W. Nazarewicz, Nucl. Phys. A410 (1983) 254.

67. P. Möller, and J.R. Nix, J. Phys. G 20 (1994) 1681.

68. R. Smolańczuk, J. Skalski, and A. Sobiczewski, Phys. Rev. C52 (1995) 1871.

69. A. Sobiczewski, F.A. Gareev, and B.N. Kalinkin, Phys. Lett. 22 (1966) 500.

70. H. Meldner, Ark. Fys. 36 (1967) 593.

71. C. Gustafson, I.L. Lamm, B. Nilsson, and S.G. Nilsson. Ark. Fys. 36 (1967) 613.

72. S. Ćwiok et al., Nucl. Phys. A611 (1996) 211.

73. K. Rutz et al., Phys. Rev. C56 (1997) 238.

74. N.V. Zamfir, G. Hering, R.F. Casten, and P. Paul, Phys. Lett. B 357 (1995) 515.

75. A. Bohr. Phys. Rev. 81 (1951) 134.

76. T.L. Khoo et al., Phys. Rev. Lett. 77 (1996) 1707.

77. A. Lopez-Martens et al., Phys. Lett. 380B (1996) 18.

78. P.H. Heenen et al., to be published.

79. M.J. Joyce et al., Phys. Rev. Lett. 71 (1993) 2176.

80. S. Bouneau et al., Phys. Rev. C53 (1996) R9.

81. H. Savajols et al., Phys. Rev. Lett. 76 (1996) 4480.

82. D. Nisius et al., Phys. Lett. 392B (1997) 18.

83. E.F. Moore $e t$ al., Phys. Rev. C55 (1997) R2150.

84. A. Bohr and B.R. Mottelson. Nuclear Structure, vol. 2 (W.A. Benjamin. New York. $1975)$.

85. V.M. Strutinsky, A.G. Magner, S.R. Ofengenden and T. Døssing. Z. Plyys. A283 (197T) 269 .

86. I. Ragnarsson, S.G. Nilsson and R.K. Sheline, Phys. Rep. 45 (1978) 1.

87. P..J. Twin. Nucl. Phys. A522 (1991) 13c.

88. R.V.F. Janssens and T.L. Khoo, Annu. Rev. Nucl. Part. Sci. 41 (1991) 321.

89. C. Baktash et al., Phys. Rev. Lett. 74 (1995) 1946.

90. C.E. Svensson et al., Phys. Rev. Lett. 79 (1997) 1233.

91. M. Freer, R.R. Betts, and A.H. Wuosmaa, Nucl. Phys. A587 (1995) 36.

92. T. Bengtsson, S. Åberg and I. Ragnarsson, Phys. Lett. 208B (1988) 39.

93. W. Nazarewicz, R. Wyss and A. Johnson, Nucl. Phys. A503 (1989) 285.

94. C. Baktash, B. Haas, and W. Nazarewicz, Annu. Rev. Nucl. Part. Sci. 45 (199.5) t8.5.

95. S. Flibotte et al., Phys. Rev. Lett. 71 (1993) 4299.

96. D.S. Haslip et al., Phys. Rev. Lett. 78 (1997) 3447.

97. I.M.Pavlichenkov, Phys. Rev. C55 (1997) 1275.

98. H. Toli and L.-A. Wu, Phys. Rev. Lett. 79 (1997) 2006.

99. G. de France, this volume.

100.G. de France, C. Baktash, B. Haas and W. Nazarewicz, Phys. Rer. C53 (1996) R1070.

101.W. Satula. J. Dobaczewski, J. Dudek and W.Nazarewicz, Phys. Rer. Lett. 77 (1996) 5182.

102.R.M. Clark et al.. Phys. Rev. Lett. 78 (1997) 1868.

103.P. Fallon. this volume.

104.S. Frauendorf. Nucl. Phys. A557 (1993) 259c. 
105.I. Hamamoto and W. Nazarewicz, Phys. Rev. C49 (1994) 2489.

106.R. Wyss and W. Satula, Phys. Lett. 351B (1995) 393.

107.J.L. Egido, L.M. Robledo and R.R. Chasman, Phys. Lett. 322B (1994) 22.

108.J. Terasaki et al., Nucl. Phys. A593 (1995) 1.

109.J. Terasaki, P.-H. Heenen, H. Flocard, and P. Bonche, Phys.Rev. C55 (1997) 1231.

110.J. Fleckner, J. Kunz, U. Mosel and E. Wust, Nucl. Phys. A339 (1980) 227.

111.U. Post, E. Wust, and U. Mosel, Nucl. Phys. A437 (1985) 274.

112.J. Dobaczewski and J. Dudek, Phys. Rev. C52 (1995) 1827.

113.J. König and P. Ring, Phys. Rev. Lett. 71 (1993) 3079. 
M98000590

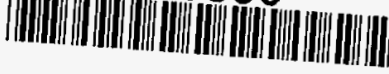

Report Number (14) ORNLICP - -94692 CONF-970622--

Publ. Date (11) 199709

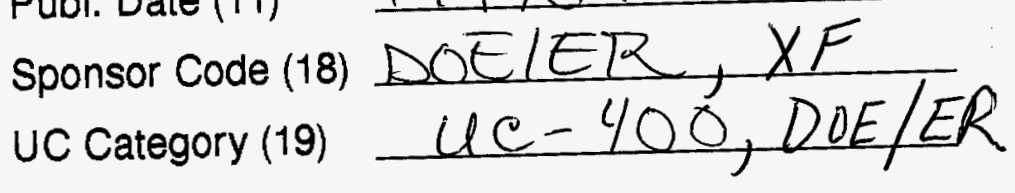

DOE 